\title{
7 Recht in der Notfallmedizin
}

\author{
Marc Nüßen
}

Historisch geht das, was als Recht bezeichnet wird, aus ethischen Grundprinzipien hervor. Das Gebot, andere stets so zu behandeln, wie man selbst behandelt werden möchte, ist eine Regel - mithin ein Rechtssatz -, der zentralen ethischen Wertvorstellungen zum praktischen Leben verhelfen soll. Waren es einst die persönlichen Vorstellungen von Sitte und Moral der herrschenden Klasse, die unmittelbar als Recht galten, entwickelte sich spätestens mit Kants Formulierung des kategorischen Imperativs eine neue Betrachtung des Verhältnisses von Ethik und Recht zueinander: „Handle stets nach einer Maxime, von der Du wollen kannst, dass sie zu einem allgemeinen Gesetz würde." Die Freiheit einer Person als Grundvoraussetzung ethischen Verhaltens kann indes die Freiheit einer anderen Person überlagern, beeinträchtigen, beschneiden. Dies macht das Aufstellen von Grenzen individueller Freiheit erforderlich. Der Staatsrechtler Georg Jellinek vertrat dem folgend die Auffassung von Recht als „ethischem Minimum“.

Die Notfallmedizin stellt ihre Akteure ständig vor Entscheidungen von hoher sittlicher oder moralischer Bedeutung. Der Umgang mit Menschen in größter Gefahr oder Not, aber auch mit Verstorbenen bringt Ausnahmesituationen mit sich im Spannungsfeld zwischen Eilbedürftigkeit und psychischer Belastung der Handelnden auf der einen Seite und dem Erfordernis ethischer Angemessenheit auf der anderen Seite. Viele der zu treffenden Entscheidungen werden jedoch anhand von Rechtsvorschriften gelenkt oder beeinflusst.

\subsection{Grundlagen}

\subsubsection{Rechtsgebiete}

Das deutsche Recht unterteilt sich grob in drei Rechtsgebiete. Das Zivilrecht, das öffentliche Recht und das Strafrecht (wobei letzteres bei näherer Betrachtung eine 
Ausprägungsform des öffentlichen Rechts ist). Das Zivilrecht regelt die Rechtsbeziehungen von natürlichen (Bürgern) und/oder privatrechtlichen juristischen Personen (Unternehmen, Vereinen etc.) untereinander. Das öffentliche Recht befasst sich mit dem Verhältnis der genannten Privatrechtssubjekte zum Staat und seinen Erscheinungsformen (in der Regel Behörden). Das Strafrecht schließlich knüpft Rechtsfolgen an bestimmte, gesellschaftlich unerwünschte Verhaltensweisen des Bürgers (Achtung: nur natürliche Personen! Unternehmen und sonstige juristische Personen, z.B. Krankenhäuser oder Rettungsdienstträger, sind im Gegensatz zu ihren Verantwortlichen nicht strafrechtsfähig).

\subsubsection{Rechtsquellen}

Hauptrechtsquelle des Zivilrechts ist das Bürgerliche Gesetzbuch (BCB). Das öffentliche Recht wird aus einer Vielzahl von Normen gespeist, die je nach grundgesetzlicher Zuständigkeitsbestimmung (Artikel 70-74 GG) von den Rechtsträgern Bund oder (Bundes)Land erlassen werden. Im Falle einer besonderen gesetzlichen Ermächtigung können öffentlich-rechtliche Normen auch von Kommunen oder bestimmten Körperschaften oder Anstalten des Bundes oder der Länder erlassen werden. Das Strafrecht ist vorrangig im Strafgesetzbuch geregelt, weitere Strafvorschriften finden sich als sogenanntes Nebenstrafrecht verteilt in zahlreichen öffentlich-rechtlichen Normen (z.B. im Arzneimittelgesetz, im Medizinproduktegesetz oder im Apothekengesetz).

\subsubsection{Rechtssystematik}

Zivilrechtliche Beziehungen entstehen in der Regel durch Vertragsschluss, also durch Vereinbarung einer Leistung und einer dafür bestimmten Gegenleistung. Hierin liegen die sogenannten Primärleistungspflichten.

Beim Behandlungs- und Transportvertrag, der typischen Vertragsart in der Notfallmedizin, besteht die Primärleistung in der Herstellung eines die Transportfähigkeit begründenden Zustands und in dem schnellstmöglichen Transport in ein geeignetes Krankenhaus. Auch die Betreuung während der Transportfahrt ist primäre Leistungspflicht (vgl. unten „Unterlassungsdelikte“). Die Kostentragung für den Einsatz (Material, Personalkosten) obliegt als primäre Gegenleistung dem Patienten bzw. dessen Krankenversicherung.

Neben den Primärleistungspflichten gibt es in jedem Vertragsverhältnis auch Schutzpflichten ( $\$ 241 \mathrm{Abs} .2 \mathrm{BCB}$ ). Jede Vertragspartei ist verpflichtet, die rechtlich geschützten Interessen der anderen Partei(en) zu achten. Eine Partei ist zum Schadensersatz verpflichtet, wenn sie im Zuge der Vertragserfüllung der anderen Partei gegenüber einen Schaden zu vertreten hat, etwa an der Gesundheit oder am Eigentum. Auch ohne ausdrückliche Vereinbarung müssen beide Seiten auf geschützte Rechtspositionen der jeweils anderen Partei Rücksicht nehmen.

Ein Behandlungs- und/oder Transportvertrag kann auf unterschiedliche Weise zustandekommen. In den meisten Fällen wird der Rettungsdienst vom Patienten zu Hilfe gerufen. Üblicherweise werden beim Zusammentreffen aber nicht die Vertragsinhalte besprochen. Sie gelten als selbstverständlich. Der Vertragsschluss wird damit stillschweigend (konkludent) vereinbart. Die Pflichten (insbesondere auch die Pflicht 
zur Kostentragung durch den Patienten bzw. dessen Krankenversicherung) entstehen in diesem Fall genau wie beim ausdrücklich vereinbarten Vertrag.

Beim nicht willensfähigen oder sogar bewusstlosen Patienten wird weder ausdrücklich noch konkludent ein Vertrag geschlossen. Hier wird der Rettungsdienst im Rahmen der Geschäftsführung ohne Auftrag ( $\$ \$$ 677ff. BCB) tätig, in der Regel als Geschäftsführung zur Gefahrenabwehr, $\mathbb{5} 680$ BGB. Maßgeblich ist der mutmaßliche Wille des Patienten. In genau diesem Umfang gilt das medizinische Personal als beauftragt und greift in den Rechts- und Interessenkreis des Patienten ein. Die Gegenleistung (Kostentragung) bemisst sich hier nicht nach einer ausdrücklichen oder vertraglichen Regelung. Stattdessen billigt das Bürgerliche Gesetzbuch gemäß $\mathbb{5} 683$ dem Rettungsdienst (als sogenanntem Geschäftsführer) einen Anspruch auf Aufwendungsersatz gegen den Patienten zu.

Wie bereits oben erwähnt, kann das Handeln bzw. Nichthandeln eines Notfallmediziners nicht nur Ansprüche des Patienten begründen. Es kann auch staatliche Sanktionierung auslösen, etwa durch strafrechtliche Verfolgung. Die Strafbarkeit bemisst sich nach drei Prüfungsebenen:

- Ist der gesetzlich normierte Straftatbestand erfüllt?

- Gibt es eine Rechtfertigung (z.B. Notwehr)?

- Ist die Tat schuldhaft verübt worden (oder bestand z.B. ein Verbotsirrtum, $\mathbb{} 17$ StCB)?

Der ärztliche Heileingriff erfüllt regelmäßig den Straftatbestand der Körperverletzung gem. $\mathbb{2} 223$ StCB, häufig sogar der gefährlichen Körperverletzung gem. $\mathbb{} 224$ Abs. 1 Zf. 1, 2 oder 5 StCB (vgl. BGH, 1 StR 319/2003). Bei Einwilligung oder (wenn diese nicht eingeholt werden kann) bei mutmaßlicher Einwilligung des Patienten besteht aber ein Rechtfertigungsgrund gem. $\mathbb{2} 228$ StCB.

Die dargestellte Grobsystematik von zivilrechtlichen Ansprüchen und von Strafbarkeitsprüfungen soll dem besseren Verständnis der weiteren Ausführungen dienen. Bei aller gebotenen Kürze handelt es sich um eine zusammenfassende Darstellung ausgewählter Rechtsbereiche, die in ganz besonderem Maße ethische Fragen in der Notfallmedizin berühren oder sich in ihrer unmittelbaren Nähe befinden.

\subsection{Pflicht zum Handeln}

Der Patient ist frei in seiner Entscheidung, ob er notfallmedizinisch behandelt bzw. transportiert werden möchte. Seine Verweigerung, sich in ein Krankenhaus befördern zu lassen, kommt in rettungsdienstlichen Situationen häufig vor. Zum Beispiel trifft der Rettungsdienst oft auf Patienten, die wegen nur geringfügiger Symptome (leichter Brustschmerz) die Ernsthaftigkeit der Ursache (Angina pectoris) unterschätzen oder sie in Eigendiagnose auf harmlose Ursachen zurückführen („Ich hab grad zu hastig ein kaltes Bier getrunken. Mit mir ist alles in Ordnung.")

Wenn ein einwilligungsfähiger Patient die Behandlung oder den Transport ernsthaft und endgültig verweigert, so muss der Rettungsdienst dies akzeptieren, auch wenn die Verweigerung allen Geboten der Vernunft zuwiderläuft. 
Auch finden sich am Einsatzort immer öfter Patientenverfügungen, in denen die Einwilligung zu bestimmten Behandlungsmaßnahmen ausdrücklich nicht erteilt wird. Das notfallmedizinische Personal hat keine vergleichbare Entscheidungsfreiheit, ob es Behandlungsmaßnahmen durchführen will. Es ist hierzu verpflichtet. Für den öffentlich-rechtlichen Rettungsdienst ergibt sich dies aus seinem hoheitlichen Sicherstellungsauftrag, für den zugelassenen privaten Unternehmer aus den Landesrettungsdienstgesetzen (z.B. $\mathbb{S} 23$ Abs. 1 RettG NRW). Unterlässt ein in der Notfallmedizin Verantwortlicher eine ihm gebotene Handlung (z.B. die Versorgung einer Wunde oder die Betreuung des Patienten während der Fahrt) und entsteht dadurch ein Schaden, löst dies in der Regel Schadensersatzansprüche aus.

Daneben kommt zumeist auch eine strafrechtliche Verantwortung in Betracht. Straftaten können nicht nur durch aktives Tun begangen werden, sondern auch durch das Unterlassen gebotener Handlungen. Es wird unterschieden zwischen echten und unechten Unterlassungsdelikten. Echte Unterlassungsdelikte sind die Straftaten, bei denen schon in der entsprechenden Strafnorm das Unterlassen als Tatbestandsmerkmal enthalten ist („Wer es unterlässt ...“), z.B. die Nichtanzeige geplanter Straftaten ( $\$ 138$ StCB) oder die unterlassene Hilfeleistung ( $\$ 323 \mathrm{C} \mathrm{StCB,} \mathrm{s.u.).}$

Regelmäßig können die sogenannten Begehungsdelikte (also der Großteil der im StCB geführten Straftaten) auch als unechte Unterlassungsdelikte begangen werden. Dies ist dann der Fall, wenn der tatbestandliche „Erfolg“ auf dem Unterlassen beruht (das Unterlassen also für das Ergebnis - etwa den Tod des Patienten - ursächlich gewesen ist) und der Unterlassende die rechtliche Pflicht hatte, dafür Sorge zu tragen, dass eben dieser „Erfolg“ nicht eintritt. Beispielsweise kann sich ein Rettungsdienstmitarbeiter eines Tötungsdelikts nicht nur durch eine aktive Tötungshandlung wie einen Schlag, einen Stich oder einen Schuss schuldig machen. Er kann auch durch das Unterlassen einer gebotenen Rettungshandlung, etwa die Verweigerung, eine schwere Blutung zu stillen, eine strafbare Tötung begehen.

Die Pflicht zur Abwendung des straftatbestandlichen „Erfolgs“ (also des Todes, der Körperverletzung usw.) wird als Garantenpflicht bezeichnet. Der Garant wird nicht nur bestraft, wenn er selbst aktiv fremde Rechtsgüter (also rechtlich geschützte Interessen eines anderen) verletzt, sondern auch, wenn er es im Rahmen seiner Möglichkeiten und Fähigkeiten nicht verhindert, dass ein Rechtsgut auf andere Weise beeinträchtigt wird (es also unterlässt). Übertragen auf die Notfallmedizin heißt das beispielsweise, dass ein Notarzt einem Patienten zum einen nicht aktiv gesundheitlichen Schaden zufügen darf, zum anderen aber auch zur Hilfe verpflichtet ist, soweit ihm diese möglich und zumutbar ist.

Eine Garantenpflicht kann aus unterschiedlichen Gründen entstehen. Sie kann zum Beispiel ausdrücklich rechtlich normiert sein, aus Lebens- oder Gefahrgemeinschaften resultieren oder aus einem pflichtwidrigen Vorverhalten begründet sein (wer eine Gefahr verursacht hat, muss dafür sorgen, dass sie sich nicht realisiert, sog. Ingerenz). In der Notfallmedizin ergibt sich die Garantenpflicht des Personals aus der Übernahme seiner Aufgaben. Der Bundesgerichtshof hat dies im Jahr 2001 folgendermaßen formuliert: „Nehmen Rettungssanitäter ihre Aufgabe wahr, entsteht ein Obhutsverhältnis gegenüber dem Betroffenen, das wesentlich von der Pflicht bestimmt ist, diesen vor weiteren gesundheitlichen Beeinträchtigungen zu bewahren (Garantenstellung durch die tatsächliche Übernahme der Gewähr für das Rechtsgut Gesundheit)." (BCH 1 StR 130/2001). 
Übrigens: Nur weil eine Person infolge ihrer Ausbildung ein besonderes Fachwissen hat (z.B. Arzt ist), entsteht keine Dauer-Garantenpflicht gegenüber jedermann. Wenn sie jedoch Hilfe leistet (z.B. in einem privat erlebten Unglücksfall), werden höhere Ansprüche an die Qualität ihrer Hilfeleistung gestellt.

Es werden zwei Formen der Garantenstellung unterschieden. Die des Beschützergaranten und die des Überwachergaranten. Der Beschützergarant steht dafür ein, dass Gefahren von einer bestimmten Person abgewendet werden. Der Überwachergarant sorgt dafür, dass sich von einer Person ausgehende Gefahren für Dritte nicht realisieren. In der Notfallmedizin kommen beide Formen der Garantenstellung in Betracht.

Als Beschützergarant sorgt der Notfallmediziner dafür, dass Gefahren vom Patienten abgewendet werden (z.B. indem er eine Blutung stillt). Als Überwachergarant verhindert er, dass der Patient gefährlich für Dritte wird (z.B. indem er einen MRSA-befallenen Patienten als solchen in der Klinik vorankündigt).

\subsection{Unterlassene Hilfeleistung}

Wie bereits oben erwähnt, ist die unterlassene Hilfeleistung ein echtes Unterlassungsdelikt. Bestraft wird, ,wer bei Unglücksfällen oder gemeiner Gefahr oder Not nicht Hilfe leistet, obwohl dies erforderlich und ihm den Umständen nach zuzumuten, insbesondere ohne erhebliche eigene Gefahr und ohne Verletzung anderer wichtiger Pflichten möglich ist. “ ( $\$ 323 \mathrm{C}$ StCB). Zweck der Vorschrift ist die Sicherung des Mindestmaßes an mitmenschlicher Solidarität innerhalb der Gesellschaft. Aus die-

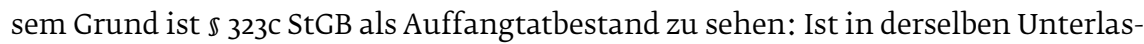
sung auch ein anderer Straftatbestand (z.B. Totschlag, Körperverletzung, Aussetzung) verwirklicht, tritt die unterlassene Hilfeleistung dahinter zurück (und wird nicht gesondert bestraft). Nur wenn das Unterlassen nicht anderweitig zu einer Strafbarkeit führt, kommt eine Bestrafung wegen unterlassener Hilfeleistung in Betracht. Somit hat $\$ 323$ C StCB eine Lückenschließungsfunktion.

Zentrale Voraussetzung ist eine spezifische Gefahrenlage, die in einem Unglücksfall, einer Lage gemeiner Gefahr oder Not bestehen kann. Unter den Begriff des Unglücksfalls im Sinne des $\$ 323$ C StCB fällt jedes mit einer gewissen Plötzlichkeit eintretende Ereignis, das eine erhebliche Gefahr bringt oder zu bringen droht (so z.B. BCH, 2 StR 63/1985). Nach BCH-Rechtsprechung sind auch Gefahrensituationen als Unglücksfall zu werten, die durch einen Selbsttötungsversuch des Gefährdeten verursacht worden sind. Es fragt sich aber, inwieweit die Abwendung der daraus resultierenden Gefahr dem Verpflichteten zumutbar ist. Bei einem freiverantwortlichen Suizidversuch kann wohl keine Hilfeleistung gegen den Willen des Betroffenen verlangt werden (Tröndle/ Fischer, $\mathbb{S} 323 \mathrm{C}, \mathrm{Rn} .5$ ). Entgegen der bisherigen BCH-Rechtsprechung entschied auch das Landgericht Deggendorf im Jahr 2013: „Eine Verpflichtung zur Vornahme von lebenserhaltenden Maßnahmen gegenüber einem freiverantwortlich handelnden Suizidenten besteht auch nicht für einen diensthabenden Notarzt. Einer entsprechenden Handlungsverpflichtung steht insofern bereits der zu beachtende Suizidwille des Patienten entgegen." (LG Deggendorf, 1 Ks 4 Js 7438/2011). Andererseits ist es für den Notarzt häufig ausgesprochen schwierig, einen frei verantwortlichen Suizidversuch von dem bewusst inkonsequenten Selbstmordversuch als „Hilferuf“ zu unterschei- 
den. Aus diesem Grund wird die Pflicht des Notarztes bei fehlender Zumutbarkeit zu verneinen sein, zugleich wird aber auch kein Verbot zum Handeln bestehen.

Eine Krankheit ist nicht per se als Unglücksfall zu werten. Nimmt diese hingegen eine plötzliche und sich rasch verschlimmernde Wendung, besteht nach herrschender Meinung ein Unglücksfall (BCH, 2 StR 63/1985), z.B. wenn sich „normale“ Herzrhythmusstörungen plötzlich zu einem Kammerflimmern umschlagen.

Unter gemeiner Not im Sinne des $\$ 323$ C StCB ist jede die Allgemeinheit betreffende Notlage zu verstehen, zum Beispiel infolge von Naturkatastrophen oder Großschadensereignissen bei Industrie-Unfällen oder Bränden (Tröndle/Fischer, $\mathbb{3} 323 \mathrm{C}, \mathrm{Rn} .8$ ).

Hilfe muss allerdings nur geleistet werden, soweit dies erforderlich, möglich und zumutbar ist. Erforderlichkeit besteht dann, wenn ohne die Hilfeleistung die Gefahr eines (ggf. weiteren oder größeren) Schadens besteht. Die Zumutbarkeit ist einzelfallabhängig. Bewertungsmaßstäbe sind insbesondere die Gefahren für den Helfenden innerhalb der Notfallsituation, aber auch seine physischen und geistigen Kräfte. Es wird einem Notarzt z.B. kaum zumutbar sein, einen Patienten aus einem brennenden PKW zu befreien. Zugleich bestehen in der Praxis häufig Konflikte, wenn der Beginn von Rettungshandlungen bis zur Sicherung der Einsatzstelle durch Feuerwehr oder Techniker warten muss (etwa bei einem einsturzgefährdeten oder brennenden Haus). Stets gilt jedoch: Das eigene Leben geht vor.

Verzichtet der Gefährdete wirksam und ausdrücklich auf Hilfe (z.B. durch Aufforderung, keine Behandlungsmaßnahmen durchzuführen oder keinen Transport in ein Krankenhaus vorzunehmen), ist das Unterlassen der Hilfeleistung nicht rechtswidrig. Auch hier bestehen jedoch oft Probleme, die Situation zutreffend zu bewerten. Schließlich ist immer denkbar, dass es sich um keinen einwilligungsfähigen Notfallpatienten handelt (z.B. infolge einer geistigen Erkrankung). Die Bewertung ist einzelfallabhängig.

Art und Maß der Hilfeleistung sind von den individuellen Fähigkeiten des Hilfspflichtigen abhängig. Notfallmedizinisches Personal wird in einem Unglücksfall am ehesten zur Hilfeleistung geeignet sein. Dies bedeutet nicht zwangsläufig, dass für

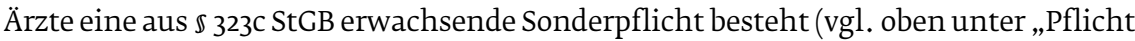
zum Handeln“). Die ärztliche Sachkunde vermag eine besondere Hilfspflicht im Sinne des $\mathbb{s} 323$ C StCB nur dann zu begründen, wenn der um Hilfe angegangene, nicht unmittelbar beteiligte Arzt wirksamere und frühere Hilfe leisten kann als andere Personen (OLG Hamm, 3 Ws 476/200o). Auch ist beim Arzt die Pflicht zur Hilfe um eine nach Lage des Falles durchzuführende Untersuchungspflicht erweitert. Die Unterlassung möglicher und zweckmäßiger Untersuchungsmethoden führt objektiv zur Erfüllung des Straftatbestands (OLG München, VII BerL 642/1977). Überweist ein praktischer Arzt einen Verunglückten aufgrund einer nur behelfsmäßig möglichen Untersuchung in ein Krankenhaus, so ist der dort als Facharzt zuständige Krankenhauschirurg hilfspflichtig (BCH, 1 StR 567/1965). Ein Arzt des Bereitschaftsdienstes macht sich hingegen wegen unterlassener Hilfeleistung strafbar, wenn er sich auf einen Anruf eines Familienangehörigen des Patienten trotz des offensichtlichen Vorliegens eines medizinischen Notfalls (plötzliches Auftreten stärkster Schmerzen bei einem Krebspatienten) weigert, den Patienten vor Ort zu untersuchen und eine ärztliche Entscheidung zu treffen und sich stattdessen z.B. auf die Empfehlung beschränkt, in eigener Entscheidung ein zweites Morphiumpflaster anzubringen (so das AG Augsburg, Cs 400 Js 123521/2003). Ein Arzt „muss bei plötzlicher Verschlimme- 
rung einer Krankheit unter Umständen eine unaufschiebbare Operation vornehmen oder veranlassen; er muss - soweit möglich - wirksame therapeutische Maßnahmen ergreifen oder dem Kranken wesentliche Erleichterung verschaffen und kann sich nicht damit begnügen, die Einweisung in ein Krankenhaus zu empfehlen. " (Tröndle/Fischer, $\$ 323 c$, Rn. 14 m.w.N.).

\subsection{Hinzuziehung der Polizei}

Die Hinzuziehung der Polizei zum Einsatzgeschehen ist notfallmedizinischer Alltag. Gleichwohl sind hierdurch rechtliche Interessen des Patienten oder seiner Angehörigen berührt, was sich sogar in strafrechtlicher Relevanz niederschlägt.

Die Weitergabe von Informationen über den Patienten an die Polizei, aber auch die Einräumung eines Zugangs zu diesen Informationen kann die Verletzung von Privatgeheimnissen im Sinne des $\mathbb{2} 203$ StCB bedeuten. Geheimnisse im Sinne dieser Vorschrift sind Tatsachen, die sich auf die Person des Betroffenen beziehen und bei denen der Betroffene ein schutzwürdiges Interesse hat, dass sie höchstens einem begrenzten Personenkreis bekannt sind oder werden. $\mathbb{} 203$ StGB erfasst die in der Notfallmedizin tätigen Berufsgruppen, insbesondere Ärzte, Notfallsanitäter (als Angehörige eines Heilberufs, der für die Berufsausübung oder die Führung der Berufsbezeichnung eine staatlich geregelte Ausbildung erfordert) und Rettungssanitäter (als Gehilfen im Sinne des $\mathbb{2 0} 3$ Abs. 3 S. 2 StCB). Eine ehrenamtliche Tätigkeit reicht aus, soweit damit eine organisatorische Eingliederung verbunden ist (Tröndle/Fischer, 59. Auflage, 2012, $\$ 203$, Rn. 21).

Eine Strafbarkeit wegen des Offenbarens kommt aber nur in Betracht, wenn es unbefugt geschieht. Befugnisse zur Offenbarung können sich aus unterschiedlichen Gründen ergeben. Bei Einwilligung, aber auch bei mutmaßlicher Einwilligung (wenn der Betroffene infolge Krankheit oder Tod zur Erteilung der Befugnis außerstande ist, zugleich aber ein offensichtliches Interesse an der Offenbarung hat/hätte) besteht eine Berechtigung zur Weitergabe der Informationen. Beispielsweise wäre es wohl nicht strafbar, nach dem Transport die Angehörigen eines unterwegs verstorbenen Notfallpatienten darüber in Kenntnis zu setzen, dass dieser mehrere Tausend Euro in bar bei sich geführt hat.

Es gibt auch gesetzliche Pflichten zur Offenbarung fremder Geheimnisse. Diese haben häufig präventiven Charakter, dienen also der Abwehr (weiterer, größerer) Gefahren. Ein Beispiel liegt etwa in einer Reihe von Vorschriften des Infektionsschutzgesetzes. Ein Geheimnisträger macht sich durch Verschwiegenheit unter Umständen sogar strafbar, nämlich wenn er von einer bestimmten geplanten Straftat erfährt, diese jedoch nicht zur Anzeige bringt ( $\$ 138 \mathrm{StCB}$ ). Zu weiteren Einzelheiten der sogenannten „Schweigepflicht“ siehe Kapitel 19.

Gerechtfertigt ist die Information des Jugendamtes oder der Polizei durch einen Arzt zum Beispiel auch bei Verdacht auf Kindesmisshandlungen und Wiederholungsgefahr (die regelmäßig naheliegt, Tröndle/Fischer, 59. Auflage, 2012, $\mathbb{2} 203$, Rn. 47). Ebenfalls gerechtfertigt ist die Verständigung der Polizei zu Zwecken des Eigenschutzes. Bedroht zum Beispiel ein HIV-Patient das Personal mit einer benutzten Kanüle, ist die Weitergabe der Information, dass der Patient „HIV positiv“ ist, an die zu Schutzzwecken herbeigerufene Polizei gerechtfertigt. 
Anders sieht es bei der Offenbarung von Geheimnissen zu repressiven Zwecken, also Zwecken der Strafverfolgung, aus. Erfährt der Geheimnisträger im Rahmen seiner Berufsausübung von begangenen Straftaten, so ist er grundsätzlich weiterhin zur Verschwiegenheit verpflichtet. Allgemeine Interessen der Strafverfolgung können die Offenbarung von Geheimnissen in der Regel nicht rechtfertigen (Tröndle/Fischer, 59. Auflage, 2012, $\mathbb{2 0 3}$, Rn. 47). Auch hier bestehen indes Ausnahmen, die zum Beispiel in den landesrechtlichen Bestattungsgesetzen geregelt sind. Beispielsweise bestimmt das Landesbestattungsgesetz Nordrhein-Westfalen in $\mathbb{9} 9$ Abs. 2 und 5, dass Notärzte bei Auffinden von Anhaltspunkten einer nicht natürlichen Todesursache (Selbsttötung, Unfall, Einwirkung Dritter) an einer Leiche oder bei sonst darauf hindeutenden Umständen unverzüglich die Polizei zu verständigen haben. Gleiches gilt für den Fall, dass die Identität eines Toten nicht festgestellt werden kann (Abs. 6).

Diese Regelungen sind Ausfluss der in $\$ 159$ Abs. 1 StPO normierten Pflicht der Polizei- und Gemeindebehörden, bei Anhaltspunkten für eine nicht natürliche Todesursache oder bei Auffindung des Leichnams eines Unbekannten eine sofortige Anzeige an die Staatsanwaltschaft oder das Amtsgericht vorzunehmen. Durch die landesbestattungsrechtliche Regelung soll eine vollständige Informationskette sichergestellt werden, bei der schnellstmöglich alle zur Beweissicherung und zur Rekonstruktion des Schadensereignisses bzw. der Straftat erforderlichen Anhaltspunkte gesichert werden können.

Aus dem oben Dargestellten lassen sich für die Hinzuziehung der Polizei folgende Faustregeln herleiten:

- Ist der einwilligungsfähige Patient das Opfer einer Straftat, darf die Polizei hierüber nur nach ausdrücklicher Einwilligungserklärung informiert werden.

- Ist der Patient nicht einwilligungsfähig (z.B. bewusstlos) oder tot und nach den äußeren Umständen möglicherweise das Opfer einer Straftat, so liegt wenn keine anderslautenden Anhaltspunkte vorliegen - eine mutmaßliche Einwilligung in die Verständigung der Polizei vor. Gibt es sogar Anhaltspunkte für eine nicht natürliche Todesursache des Patienten, besteht u.U. nach Landesrecht die Pflicht des Arztes, die Polizei zu verständigen (vgl. Bestattungsgesetze der Länder).

- Ist der Patient tot und ist seine Identität unklar, besteht u.U. ebenfalls die landesrechtliche Pflicht, die Polizei zu verständigen.

v Ist der Patient nach den äußeren Umständen Täter einer Straftat, so darf die Polizei nur verständigt werden, wenn dies zur Verhinderung weiterer Straftaten erforderlich ist. Das Ziel der Bestrafung des Täters ist kein zureichender Grund.

\subsection{Beschlagnahme und Freigabe von Leichen}

Sind Anhaltspunkte dafür vorhanden, dass jemand eines nicht natürlichen Todes gestorben ist, oder wird der Leichnam eines Unbekannten gefunden, sind die Polizei- und Gemeindebehörden zur sofortigen Anzeige an die Staatsanwaltschaft oder an das Amtsgericht verpflichtet, $\mathbb{\$} 159$ Abs. 1 StPO. Zugleich darf in einem solchen Fall die Bestattung nicht ohne schriftliche Genehmigung der Staatsanwaltschaft erfolgen, $\mathbb{\$} 159$ Abs. 2 StPO. Hieraus leitet sich für die Polizei das ungeschriebene Gebot ab, zur Sicherung von Ermittlungen unverzüglich für die Bergung und Bewachung 
oder sichere Unterbringung der Leiche zu sorgen (Meyer-Goßner, $\mathbb{1} 159$, Rn. 4). Ihre Überführung von der Unfallstelle bzw. vom Tatort oder sonstigen Fundort an einen anderen Ort kann die Polizei vornehmen, wenn sie zuvor hinreichende Bemühungen zur Beweissicherung (Fertigung von Fotos der Leiche am Fundort, Augenscheinnahme durch Staatsanwalt oder Richter usw.) angeregt oder durchgeführt hat.

\section{Die Beweissicherung tritt hinter die Rettungsbemühungen der Notfallmedizin zurück. Zunächst muss alles getan werden, um das Leben des Patienten zu retten. Anschließend (ggf. nach nicht erfolgreichen Rettungsbemühungen) kommt die Beweissicherung in Betracht.}

Sollte der Rettungsdienst vor der Polizei eintreffen und einen offensichtlichen Todesfall vorfinden, bei dem keine Reanimationshandlungen mehr geboten sind, sollte er das Eintreffen der Polizei abwarten (bzw. diese zunächst verständigen) und die Einsatzstelle bis dahin wie vorgefunden belassen. Macht dies längere Wartezeiten erforderlich, sollte das Warten im Zustand der Einsatzbereitschaft durchgeführt und abgebrochen werden, sobald ein neuer Notfalleinsatz das Verlassen der Einsatzstelle erforderlich macht.

Gegenstände, die als Beweismittel für die Untersuchung einer Straftat von Bedeutung sein können, sind von den Ermittlungsbehörden in Verwahrung zu nehmen oder in anderer Weise sicherzustellen, $\mathbb{} 94$ Abs. 1 StPO. Zu diesen „Gegenständen“ gehören auch Leichen (Meyer-Goßner, $\mathbb{5}$ 94, Rn. 4). Wird eine Leiche, die potenzielle Beweisbedeutung hat, von den Angehörigen nicht freiwillig herausgegeben, bedarf es gem. $\mathbb{S} 94$ Abs. 2 StPO der Beschlagnahme. Die Beschlagnahme ist die förmliche Sicherstellung eines Gegenstands durch Überführung in ein amtliches Verwahrungsverhältnis. Sie erfolgt auf gerichtliche Anordnung. Bei Gefahr im Verzug kommt auch die Anordnung der Staatsanwaltschaft oder der Polizei in Frage ( $\$ 98$ Abs. 1 StPO i.V.m. $\$ 152 \mathrm{GVG}$ ). Im letzteren Fall ist regelmäßig binnen drei Tagen eine nachträgliche gerichtliche Bestätigung zu beantragen ( $\$ 98 \mathrm{Abs} .2 \mathrm{StPO})$.

Mit Abschluss des Verfahrens endet die Beschlagnahme (ohne förmliche Aufhebung der Beschlagnahmeanordnung). Die Aufhebung der Beschlagnahme ist aber auch dann schon geboten, sobald die beschlagnahmten Gegenstände nicht mehr für die Zwecke des Strafverfahrens benötigt werden (vgl. BCH 3 ZR 116/1977). Nach Aufhebung der Beschlagnahme sind die Gegenstände grundsätzlich an den letzten Gewahrsamsinhaber herauszugeben (BCH 3 ZR 116/1977, Zf. 75 Abs. 2 RiStBV). Die Herausgabe ist Sache der Staatsanwaltschaft (Zf. 75 RiStBV). Die Transportkosten im Zuge der Beschlagnahme (jedenfalls für den Weg in die amtliche Verwahrung) trägt das Bundesland, deren Gericht oder Staatsanwaltschaft die Beschlagnahme der Leiche veranlasst hat, es sei denn, es ist landesgesetzlich ausdrücklich etwas anderes geregelt. Dient der Transport dem Zweck, die Leiche zur Klärung der Todesursache zu sichern, nimmt das Land ein objektiv eigenes Geschäft auf dem Gebiet der Strafrechtspflege wahr (vgl. OVG Berlin-Brandenburg, $12 \mathrm{~N}$ 12/2013).

Ein Transport von Leichen mit Rettungsmitteln ist grundsätzlich nicht zulässig. 
Je nach landesgesetzlicher Regelung kommt ein solcher Transport zum einen nur in einem für diesen Transport geeigneten dicht verschlossenen Behältnis in Frage (z.B. NRW), zum anderen sind dafür besonders eingerichtete Fahrzeuge, also Leichenwagen zu verwenden (z.B. Sachsen-Anhalt). Danach wäre streng genommen auch der Weitertransport eines während der Fahrt verstorbenen Patienten unzulässig. Stellt man auf die Schutzrichtung der Landesregelungen ab (kein „offener“ Transport von Leichen im Straßenverkehr), wird man wohl zu dem Ergebnis kommen, dass der Weitertransport möglich sein muss. Immerhin ist die Belastung für die übrigen Straßenverkehrsteilnehmer ungleich größer, wenn die „Übergabe“ der Leiche vom Rettungsdienst auf den Bestatter mitten im laufenden Straßenverkehr stattfindet. Die Zulässigkeit des Weitertransports dürfte sich schon aus Gründen der Diskretion ergeben. Allerdings handelt es sich hierbei um ein seltenes Problem, da die Reanimation nur in den seltensten Fällen „unterwegs“ abgebrochen wird, sondern ihr Ende üblicherweise am Einsatzort oder in der Notaufnahme des Krankenhauses findet.

\subsection{Leichenschau}

Der Erlass von Gesetzen, die Bestattungs- und Friedhofsangelegenheiten regeln, ist gemäß Art. 70 Abs. 1 GG Sache der Länder. Die Landesbestattungsgesetze weisen zum Teil deutliche Unterschiede auf. So gilt beispielsweise in Schleswig-Holstein als Leiche „der Körper eines Menschen, bei dem sichere Todeszeichen bestehen oder der Tod auf andere Weise zuverlässig festgestellt wurde und bei dem der körperliche Zusammenhalt durch den Verwesungsprozess noch nicht vollständig aufgehoben ist." In Sachsen-Anhalt gilt zusätzlich auch das Skelett eines Menschen als Leiche.

Die Regelungen zu Durchführungspflicht, Veranlassung, Ort und Zeit der Leichenschau decken sich bundesweit aber überwiegend. Jeder niedergelassene Arzt ist wenn verfügbar - für die Feststellung des Todes, des Todeszeitpunkts, der Todesart und der Todesursache eines Verstorbenen unverzüglich zur Durchführung der Leichenschau verpflichtet. Zur unverzüglichen Verständigung des Arztes verpflichtet sind Personen, in deren Beisein der Tote verstorben ist bzw. die den Toten aufgefunden haben (z.B. Schleswig-Holstein, Sachsen-Anhalt) oder die Angehörigen des Toten (z.B. Nordrhein-Westfalen) oder die Personen, in deren Wohnung, Einrichtung oder auf deren Grundstück sich der Sterbefall ereignet hat (z.B. Baden-Württemberg).

\footnotetext{
In der Regel sind Notärzte von der Verpflichtung zur Durchführung der vollständigen Leichenschau befreit. Ihre Pflicht beschränkt sich auf die Ausstellung einer „vorläufigen Todesbescheinigung".
}

Ein Notarzt, der keine vollständige Leichenschau durchführt, sollte die Angehörigen aber darauf hinweisen, dass sie hierfür einen anderen Arzt herbeizurufen verpflichtet sind. Unter Umständen kann sich aber auch für den Notarzt eine Pflicht zur Durchführung der vollständigen Leichenschau ergeben. In dem Fall, in dem kein Arzt der Kassenärztlichen Vereinigung verfügbar ist, begründet sich z.B. gem. $\$ 9$ Abs. 3 BestC NRW eine Zuständigkeit der unteren Gesundheitsbehörde. Auf kommunaler Ebene kann wiederum geregelt sein, dass das Gesundheitsamt vom Notarzt vertreten wird. Wenn in einem solchen Fall der Notarzt die Leichenschau durchführt, muss jedoch 
dauerhaft die Einsatzbereitschaft für einen Folgeeinsatz bestehen (nähere Darstellung des Themas bei Madea, Zf. 5.1).

Gegenstand der Leichenschau ist in der Regel die umfassende und sorgfältige Sichtung der unbekleideten Leiche. Im Anschluss ist die Todesbescheinigung auszufüllen und auszuhändigen. Diese besteht aus einem nichtvertraulichen (für das zuständige Standesamt bestimmten) und einem vertraulichen Teil (der an das zuständige Gesundheitsamt adressiert ist). Im nichtvertraulichen Teil sind die Angaben zur Identifikation der Leiche einschließlich der bisherigen Anschrift, des Zeitpunkts, der Art und des Orts des Todes und bei möglicher Gesundheitsgefährdung eines Warnhinweises enthalten. Im vertraulichen Teil finden sich insbesondere Angaben zurTodesfeststellung, zur Todesursache und zu den weiteren Umständen des Todes.

Verpflichtete, die nicht unverzüglich die Leichenschau veranlassen, sowie Ärzte, die die Leichenschau nicht unverzüglich oder nicht sorgfältig durchführen oder trotz Anzeichen einer nicht natürlichen Todesursache die Polizei oder die Staatsanwaltschaft nicht verständigen, begehen eine Ordnungswidrigkeit (z.B. $\mathbb{1} 19$ Abs. 1 Zfn. 2 und 3 BestG NRW). Im Falle der nicht sorgfältig durchgeführten Leichenschau befreit sich der Arzt auch nicht mit dem ausdrücklichen Hinweis in der Todesbescheinigung, die Leichenschau nicht mit der erforderlichen Sorgfalt durchgeführt zu haben.

In der Praxis besteht häufig das Bedürfnis, das weitere Prozedere zu beschleunigen oder zu erleichtern. Nicht selten wird auf den die Leichenschau durchführenden Arzt dann „Druck von außen“ ausgeübt, in der Todesbescheinigung eine „natürliche Todesursache“ anzugeben, obwohl es Anzeichen einer nicht natürlichen Todesursache gibt. Dies kann für den Arzt zu einer Strafbarkeit wegen Falschbeurkundung im Amt ( $\$ 348 \mathrm{StCB})$, wegen mittelbarer Falschbeurkundung (\$ $271 \mathrm{StCB})$ und/oder wegen (versuchter) Strafvereitelung ( $\$ 258 \mathrm{StCB}$ ) führen.

\section{Wenn der Arzt Anzeichen für eine nicht natürliche Todesursache erkennt, sollte er dies unbedingt korrekt dokumentieren und sich nicht beeinflussen lassen.}

Dies ist er dem Verstorbenen, der Rechtspflege und nicht zuletzt sich selbst schuldig.

\subsection{Sektion}

Rechtliche Maßgaben für die Durchführung der Sektion sind sowohl im Bundes- als auch im jeweiligen Landesrecht zu finden. Grundsätzlich kann zwischen drei Typen von Sektionen unterschieden werden (s. Tab. 11). Andere Fälle (z.B. die Sektion zur Bekämpfung von Infektionskrankheiten gem. $\$ \$ 25,26$ IfSchG) sollen an dieser Stelle nicht näher betrachtet werden.

Die rechtsmedizinische Sektion wird vom Richter, in dessen Bezirk sich die Leiche befindet, oder (im Fall der Gefährdung des Untersuchungserfolgs bei Verzögerung) von der Staatsanwaltschaft angeordnet, $\mathbb{5} 87 \mathrm{Abs}$. 4 StPO. Die vorherige Zustimmung des Verstorbenen zu Lebzeiten oder nunmehr seiner Angehörigen ist nicht erforderlich, ein ausdrücklicher Widerspruch irrelevant. Es kommt allein auf die Erforderlichkeit der Sektion an. Erforderlich ist sie, wenn fremdes Verschulden am Tod in 
Tab. 11 Typen von Sektionen

\begin{tabular}{l|l}
$\begin{array}{l}\text { Rechtsmedizinische } \\
\text { Sektion }\end{array}$ & $\begin{array}{l}\text { Im Rahmen eines strafrechtlichen Ermittlungsverfahrens ist zu klären, ob der Tod } \\
\text { Folge einer Straftat gewesen ist oder ob die Leiche Hinweise darauf liefert, wie eine } \\
\text { Straftat begangen worden ist bzw. wer der Täter gewesen sein könnte. }\end{array}$ \\
\hline Klinische Sektion & $\begin{array}{l}\text { Die Todesursache ist festzustellen mit dem Ziel, die Qualität der ursprünglichen } \\
\text { Diagnose oder der darauf gefolgten Therapie zu bewerten. }\end{array}$ \\
\hline Anatomische Sektion & Zur Ausbildung von Medizinstudenten wird der Körper eines Verstorbenen obduziert. \\
\hline
\end{tabular}

Betracht kommt und die Todesursache oder Todeszeit festgestellt werden muss (BVerfG, 2 BvR 1912/1993). Bloße Anhaltspunkte für einen nicht natürlichen Tod ( 159 StPO) reichen demnach nicht aus. Die Sektion nehmen zwei Ärzte vor, die beide ununterbrochen anwesend sein müssen ( $\$ 87 \mathrm{Abs} .2 \mathrm{StPO}$ ).

Zur klinischen und zur anatomischen Sektion finden sich Regelungen meist in den Bestattungsgesetzen der Länder. Hamburg und Berlin haben gesonderte Sektionsgesetze. In der Regel ist die ausdrückliche Zustimmung des Verstorbenen zu Lebzeiten erforderlich.

\subsection{Organentnahme bei Unfallopfern}

Zur Organentnahme beim toten Spender treffen $\mathbb{\$}$ 3-7 des Transplantationsgesetzes die zentralen Regelungen. Das Transplantationsgesetz ist allerdings nicht (unmittelbar) einschlägig, wenn eine Gewebeentnahme nicht zum Zwecke der Übertragung auf einen anderen Menschen bestimmt ist, sondern reine Sektionshandlung ist (also z.B. der Rekonstruktion des Unfallhergangs dient). Toter im Sinne des $\mathbb{3} 3 \mathrm{Abs}$. 2 TPC ist, wessen Hirntod nach dem Stand wissenschaftlicher Erkenntnis entsprechender Verfahrensregeln festgestellt ist. Diesen Stand hält die Bundesärztekammer in ihren Richtlinien fest, $\mathbb{1} 16$ Abs. 1 Zf. 1 TPG.

Die Entnahme ist grundsätzlich nur zulässig, wenn der Spender zuvor eingewilligt hatte. Wenn er ausdrücklich widersprochen hatte, kommt die Entnahme nicht in Frage. Liegt keine ausdrückliche, schriftliche Einwilligung (bzw. kein Widerspruch) vor, kann unter den Voraussetzungen des $\mathbb{4} 4$ TPG der nächste Angehörige des Verstorbenen befragt werden. Wenn auch diesem keine Entscheidung des Verstorbenen bekannt ist, kann die Entscheidung des Angehörigen selbst für den Verstorbenen eingeholt werden. Seine Zustimmung führt zur Zulässigkeit der Organentnahme (sog. erweiterte Zustimmungslösung). Es handelt sich hierbei nicht um seine eigene Entscheidung, sondern um eine Entscheidung auf Crundlage der dem Angehörigen bekannten Ansichten und Moralvorstellungen des Verstorbenen. Der Angehörige ist zu dieser Entscheidung nur befugt, wenn er innerhalb der letzten zwei Jahre Kontakt zum Verstorbenen hatte. Dies ist von dem Arzt zu erfragen der die Organentnahme durchführt. Cibt es mehrere Angehörige gleichen Ranges, so genügt zur Entscheidungsfindung die Einbindung nur eines Angehörigen. Der Widerspruch eines von mehreren Angehörigen des obersten Rangs genügt jedoch, die Organentnahme zu verhindern. Ist ein Angehöriger des obersten Rangs nicht in angemessener Zeit erreichbar, so kann die Entscheidung durch den zuerst erreichbaren nächsten Angehörigen getroffen werden. 


\section{Literatur}

Fischer T (2012) Strafgesetzbuch. 59. Aufl. Beck, München

Madea B (2013) Die ärztliche Leichenschau. 3. Aufl. Springer, Berlin/Heidelberg

Meyer-Goßner L, Schmidt B (2012) Strafprozessordnung. 55. Aufl. Beck, München

\section{Zur Vertiefung empfohlen}

Hörnle T (2010) (Straf-)Rechtliche Aspekte der Sektion nach deutschem Recht. In: Tag B, Groß D (Hrsg.) Der Umgang mit der Leiche. Campus Verlag, Berlin/New York

Wainwright U (2012) Die Leichenschau - Fallstricke und Irrtümer. In: Der Allgemeinarzt 12: 38ff.

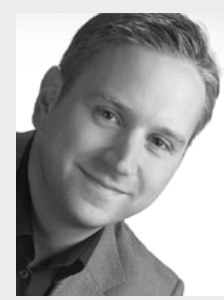

\section{Ass. iur. Marc Nüßen}

Studium der Rechtswissenschaft in Bochum. Rechtsreferendariat in Bochum und Speyer. Ab 2000 ehrenamtliche Tätigkeit als Rettungssanitäter. Seit 2005 nebenberuflich Dozent für „Rechtskunde im Rettungsdienst“, diverse juristischnotfallmedizinische Veröffentlichungen (u.a. „www.recht-im-rettungsdienst.de“, Co-Autor „Notfallmedizin“ im Verlag akaMedica) und Vorträge (u.a. DKOU 2012). 